Document downloaded from:

http://hdl.handle.net/10251/153119

This paper must be cited as:

Boza, A.; Alarcón Valero, F.; Alemany Díaz, MDM.; Cuenca, L. (2017). Event Monitoring System to Classify Unexpected Events for Production Planning. Lecture Notes in Business Information Processing. 291:140-154. https://doi.org/10.1007/978-3-319-62386-3_7

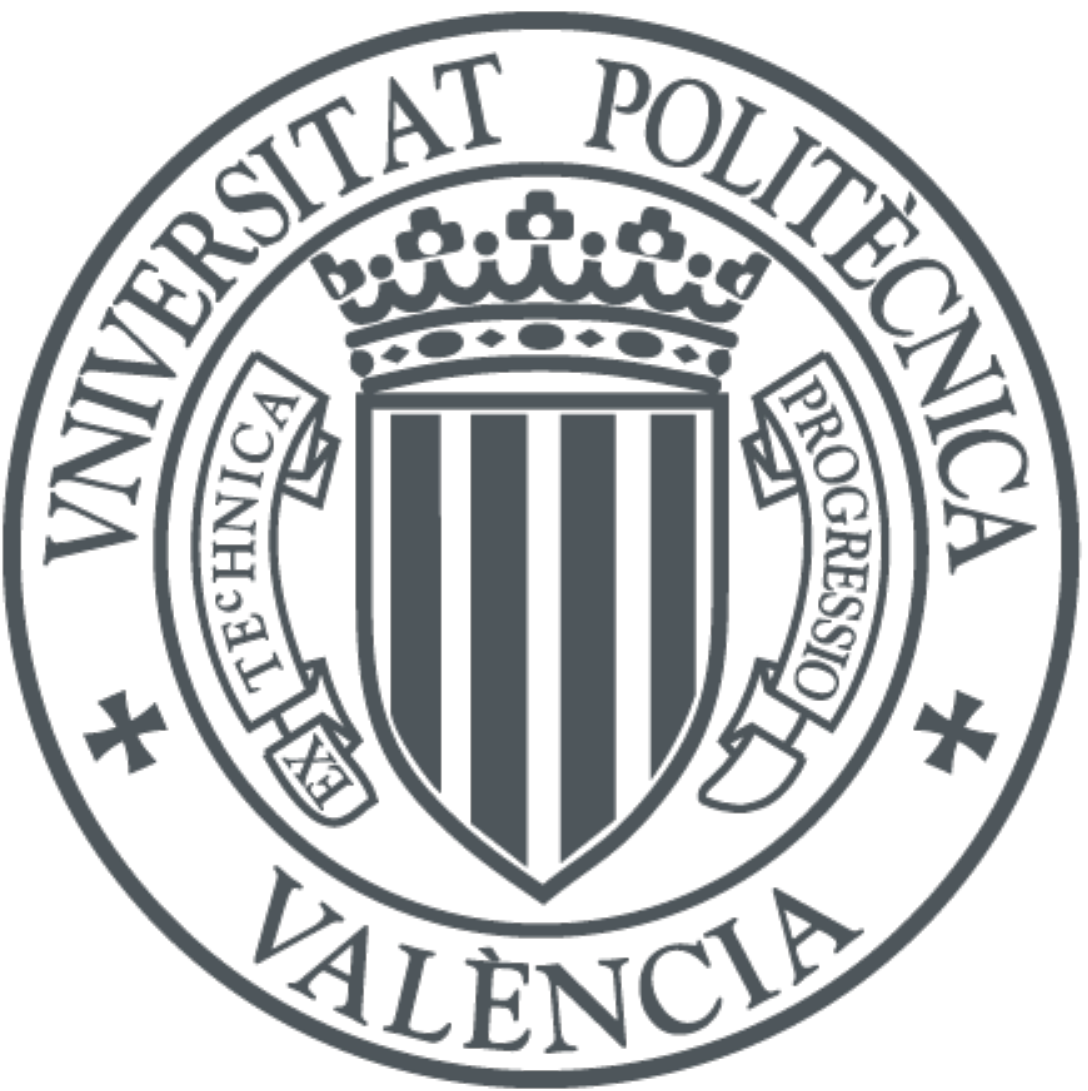

The final publication is available at

https://doi.org/10.1007/978-3-319-62386-3_7

Copyright Springer-Verlag

Additional Information 


\title{
Event Monitoring System to Classify Unexpected Events for Production Planning
}

\author{
Andrés Boza, Faustino Alarcón, M.M.E. Alemany, Llanos Cuenca \\ Research Centre on Production Management and Engineering (CIGIP) \\ Universitat Politècnica de València, Valencia, Spain \\ \{aboza, faualva, mareva, llcuenca\}@cigip.upv.es
}

\begin{abstract}
Production planning prepares companies to a future production scenario. The decision process followed to obtain the production plan considers real data and estimated data of this future scenario. However, these plans can be affected by unexpected events that alter the planned scenario and in consequence, the production planning. This is especially critical when the production planning is ongoing. Thus providing information about these events can be critical to reconsider the production planning. We herein propose an event monitoring system to identify events and to classify them into different impact levels. The information obtained from this system helps to build a risk matrix, which determines the significance of the risk from the impact level and the likelihood. A prototype has been built following this proposal.

Keywords: Production Planning, Event Management, Decision Making, Information System.
\end{abstract}

\section{Introduction}

The production planning is one of the key functions in a company. Planning deals with finding plans to achieve some goal [1] and production planning is a partial planning approach for a particular function of a company [2]. Production planning also usually covers the allocation of activities to factory departments, which is a typical scheduling task. Production planning uses information to generate processing routes and to find what raw material should be ordered and when [1]. The production planning basically involves finding the most efficient way to use production resources in order to fulfill the demand requirements with regard to quality, quantity and delivery date.

Once production-planning decisions have been made and planning is ongoing, unexpected events can appear. Any cause (e.g. machine breakdowns or changes in firm orders) that endangers current production plan validity could lead to regenerating the entire plan [3]. However, making a new plan can be complex and time consuming when there is a lot of information to use (big bill of materials or a great variety of products). But, the main difficulty is to adapt the ongoing production plans, which produces that often no changes are made [4]. The conception and implementation of appropriate information and communication systems is a basic 
condition for identifying critical incidents [5]. In this sense, Sacala et al. [6] indicate that data collected from sensors must trigger a chain of events leading to changes within enterprise business process, collaboration mechanism or organizational framework. Such changes can be achieved in terms of simple sense-act enterprise behaviour (direct link between sense and act) or more complex sense-plan- act approach (decision level). Hence the first objective of an event monitoring system is to sense production information about a real-time environment and to detect events.

Enterprises normally use tools that provide them with information to make decisions. According to [7], Decision Support Systems (DSSs) are designed to use decision makers' own insights and judgments in an ad hoc, interactive analytical modeling process, which leads to a specific decision. So an event monitoring and management system should interact with DSSs to manage events that might affect previously made decisions. It should act as a supra-system that gather the necessary information to identify when previous decisions are still valid or need to be reanalyzed. Thus traditional DSS configuration should be extended to treat event management by a monitoring and management system, which monitors internal and external information [8]. This event information can also be represented in the form of rules, such as IF-THEN. These rules include events (or signals) that can alter the plans (IF...) and also the warning signal in each case (...THEN...). For example, IF a priority and very important customer order comes AND the production planning is just launched THEN a warning signal must be trigged, which may advise re-planning. This set of rules represents an expert system: it contains information obtained from a human expert, which is represented in form of rules [9].

According to the ISO/Guide 73:2009 [10], risk is the combination of the probability of an event and its consequences when exploiting any vulnerability. So, once events are identified, the associated risks can also be estimated. We propose herein a monitoring software application, based on rules, that detects unexpected events in production planning and identify risks produced by these events. In order to explain our purpose in this paper: Section 2 reviews problems in production planning in the literature; Section 3 deals with event management; Section 4 defines expert decision support system based on the literature; Section 5 explains our proposal to monitor and classify events; Section 6 offers a prototype of this proposal; Section 7 presents the conclusions drawn from this approach.

\section{Incidences in Production Planning}

The occurrence of certain unexpected events or incidences, for example, a broken machine or a huge order may invalidate the ongoing production plan. In the literature, authors have dealt with these problems in different ways. Chan et al. [11] indicate that frequent changes in the current schedule may lead to disturbances in production, and may result in lateness orders or increased production costs. Weinstein and Chung [12] explain that when production equipment displays signs of failure, or they occur after, this may adversely affect both production plan integrity and product quality. Poon et al. [13] explain that in the actual manufacturing environment, shop floor managers 
face numerous unpredictable risks in day-to-day operations, such as defects in supplies of components or raw materials, or errors, failures and wastage in various production processes. Baron and Paté-Cornell [14] indicate that during the manufacturing process, unexpected interruptions appear, which could be accidents, machine breakdowns or human errors. In a cookie factory case, Van Wezel et al. [4] study planning flexibility and classify events according to their source: a) Customer (e.g. rush order, change in order volume, or earlier/later delivery date); b) Product (e.g. raw material out of stock, too little or too much stock of end product(s), or product sent back); c) Process (e.g. setup/cleaning time variation, more/less waste, or higher/ lower production speed; d) Machines/staff (e.g. long disruptions, shortage or surplus capacity, or variation in run-in times). All these planning problems need to be managed and it is necessary to decide how to deal with these events. The objective is to minimize the impact caused in the whole company.

A proper management of these problems requires an identification and enumeration of them, including a study of where, how and when can appear. Furthermore, their detection is a very important task. If the detection of the event is slow, the troubles will be bigger. In this sense, new technologies based on the Industry 4.0 concept like Internet of Things can help in this purpose. Once an event occurs in a company, event information is stored in the system and analysis information is delivered. With this information, decision-makers decide what action must to take to solve the problem A quicker identification of relevant events is necessary to make a quicker analysis of their consequences. SAP [15] highlights how value diminishes as time elapses between when data is first captured and when an action or decision is triggered. Of course, this analysis must include not only a short-term point of view, but also the consequences for the ongoing production planning.

\section{Event Management}

Shamsuzzoha et al. [5] state that an event can be defined as an incident or occurrence that might evolve from either internal or external sources of operations within the network. An event can be identified assessing if a deviation of the current status as compared the planned one exists. Events should be viewed on a real-time basis. For achieving this, automated event-detection systems are usually necessary. But an event monitoring is more than an event-detection system. Boza et al. [8] indicate that an event monitoring system is a part of an event management system. Event management provides systems with a proactive response to business events, anticipating and planning solutions before damage is produced.

The literature includes various authors who deal with event management not only for a company but also for business networks, such as Virtual Organizations [16] or Collaborative Networks [17]. Baron and Paré-Cornell [14] provide an analytical and dynamic link between the Risk Management System and the long-term productivity and safety performance of the physical system. Barash et al. [18] propose a decision support tool for the business impact analysis and improvement of the incident management process in IT support organization. Bartolini et al. [19] present an 
approach to assess and improve the performance of an IT support organization in managing service incidents based on the definition of a set of performance metrics and a methodology. This guided analysis allows users to find the root causes of poor performance and to decide about the corrective actions to be taken. Liu et al. [20] develop an approach for modeling event relationships in a supply chain through Petri nets as a formalism for managing events. Söderholm [21] aims to outline different categories of unexpected events that appear in projects as a result of environmental impacts and how these are dealt with. Bearzotti et al. [22] present an agent-based approach for the Supply Chain Event Management problem, which can perform autonomous corrective control actions to minimize the effect of deviations in the plan currently underway.

The impact of an event can be positive or negative, representing the last one a risk. Events implying some risks are priority to be notified with the aim of their properly management assessment and response. The urgency of an event conditions the event notification process. This aspect leads to the necessity of classifying events in order to manage the unexpected events. Distinct classifications based on different criteria can be found in the literature: according to its impact [23], according to its supporting [24] and according to specific groups given by the company [20], [22]. Only one of these research made a monitoring system to detect events [22]. But all these approaches require an expert engineer to define the rules.

A very accepted classification of events is according to their impact in the organization on a scale from 1 to 5 , where 1 represents the least level and 5 the strongest [5]. Knowing the severity of the event, risk can be identified by the occurrence likelihood of this event. Thus a risk matrix can be used to classify events. This matrix has several categories, "probability," "likelihood" or "frequency", for its columns and several categories, "severity," "impact" or "consequences", for its rows. It associates a recommended level of risk, urgency, priority or management action with each row-column pair; that is, with each cell [25].

These risk matrices have been widely praised and adopted as simple effective approaches to risk management. According to Cox [25], their main advantages are that they provide: (1) a clear framework for the systematic review of individual risks and portfolios of risks; (2) convenient documentation for the rationale of risk rankings and priority setting; (3) relatively simple inputs and outputs, often with attractively colored grids; (4) opportunities for many stakeholders to participate in customizing category definitions and action levels; (5) opportunities for consultants to train different parts of organizations on "risk culture" concepts at different levels of detail. So the risk matrix is an appropriate tool to classify events.

\section{Expert Decision Support System}

DSSs are normally used as a tool to make decisions when faced with certain problems. They are defined as computer systems that deal with a problem where at least some stage is semi-structured or unstructured. A computer system can be developed to deal with the structured portion of a DSS problem, but decision makers' 
judgment must consider the unstructured part, to hence constitute a human-machine problem-solving system [26]. The primary purpose of DSSs is to help decisionmakers develop an understanding of the ill-structured complex environment represented by the model [27].

When an organization has a complex decision to make or a problem to solve, it often turns to expert for advice. The experts it selects have specific knowledge about and experience in the problem area. Expert systems attempt to mimic human experts' problem-solving abilities [28]. Turban and Watkins [29] described the Expert System like a computer program, which includes a knowledge base that contains an expert's knowledge for a particular problem domain, and a reasoning mechanism for propagating inferences on the knowledge base. The benefits generated by expert systems include [30]: (1) less dependence on key personal; (2) facilitating staff training; (3) improving the quality and efficiency of decision making; (4) transferring the ability of making decisions. Integrating an Expert System into DSSs helps obtain more benefits. These benefits can be used in several dimensions [29]: Expert Systems contribution, DSS contribution, and the synergy resulting from the DSS/ES combination.

\section{Proposal of an Event Monitoring System to Classify Unexpected Events for Production Planning}

Given the advantages of the Expert DSS presented in the previous section, we propose an Event Monitoring System (EMS) based on an Expert DSS, which identifies and classifies events (CE) that have an impact on ongoing production planning and interact with the DSS used in production planning (PP) systems, dubbed as EMS-CE-PP. Expert knowledge is necessary to identify and classify potential events by their impact level. Depending on its likelihood and impact level, the system indicates the seriousness of the event in the previously shown standard risk matrix. This likelihood can be estimated by the system, counting the number of times that an event appears.

The proposed expert DSS does not use an Expert System like an intelligent program, which automatically makes a decision, but uses it like a support system for decision makers.

\subsection{Event Monitoring System (EMS) Framework}

Some enterprises generate their production planning with DSSs that use mathematical models (Model-Driven DSS). The decisions made with these Model-Driven DSSs can be affected by different events. A significant set of events to be identified includes those that affect the planning generated by these Model-Driven DSSs. The mathematical models used in these DSSs are written in modeling languages, such as Modeling Programming Language (MPL). So it is possible to extract parameters and decision variables from these models that can be affected by events. The parameters and decision variables form a set of attributes of the models. 
This is the starting point for our proposal, where an expert in production planning systems selects the set of attributes that require a control. These attributes will be used to make rules. A rule is a condition defined by the decision maker to identify the events: if this condition goes into effect, an event alert appears. These rules are made by the expert, a person with high knowledge about event detection in production. This expert is usually the decision maker.

The objective of these rules is to identify changes in the production system to reconsider the current production planning generated by the DSSs between each replanning period. The current information about the production systems can be significantly different from the previous information used by the DSSs when the current planning was generated. Ultimately, the objective is to know if the ongoing production planning is still valid or it is necessary a new production planning before its term.

This proposal extends the DSS proposed by Boza et al. [31] [32], which includes three phases: (1) model and attributes selection: experts select decision models and the attributes (of these models) that can be affected by events; (2) criteria creation and visualization: experts create alert criteria about previously selected attributes; (3) execution: validation of the alert criteria conditions executed manually or automatically. Our proposal herein extends the previous proposal to include the event classification and risk identification based on the risk matrix. This information allows the decision-maker reconsider the current production planning. The following paragraphs review these phases and detail our proposal.

\subsection{Model and Attributes Selection}

An expert in production planning systems selects the mathematical models used in the planning production decision system to analyze the alert criteria on them. After selecting the models, experts can identify the model's parameters and decision variables to create the alert criteria to identify events. These selected attributes must have impact into the production planning and its variation can produce a modification in the production decisions. For example: variation in demand or machine setup times.

\subsection{Criteria Creation and Visualization}

Alert criteria can be defined according to the selected attributes and a classification of the events can be made. We propose using five impact levels for each criterion: Extremely Serious Level, Serious Level, Substantial Level, Moderate Level and Low Level. Each level is achieved according to a logical operation formed by constants, attributes and functions. Alerts are triggered when a true value appears in these logical operations. Constants are values that are introduced directly by the expert; attributes are the previously selected parameters and decision variables; functions are operations formed by attributes and constants, such as addition, averages, etc.

Enterprise information is dynamic, so any unexpected development of an attribute should be analyzed. In order to consider this development in the alert criteria, it is 
necessary the current and/or previous values for each attribute in the alert criteria; i.e., attributes values are taken from the current production system state and/or from the previous state (when the production planning was made). Thus, decision makers introduce rules (using logical conditions) to identify events. Table 1 shows combinations in these logical conditions (A -logical condition- B).

Table 1. Possible combinations of logical operation to criteria creation.

\begin{tabular}{|c|c|c|}
\hline A & & B \\
\cline { 1 - 1 } $\begin{array}{c}\text { Current Attribute } \\
\text { Value }\end{array}$ & & $\begin{array}{c}\text { Previous Attribute } \\
\text { Value }\end{array}$ \\
\cline { 1 - 1 } $\begin{array}{c}\text { Current or Previous } \\
\text { Attribute Value }\end{array}$ & Logical & Constant Value \\
\cline { 1 - 1 } $\begin{array}{c}\text { Current or Previous } \\
\text { Attribute Value }\end{array}$ & condition & Function Result \\
\hline Function Result & & Constant Value \\
\hline Function 1 Result & & Function 2 Result \\
\hline
\end{tabular}

Alert criteria can also be defined for particular objects (e.g. the demand limit value of a specific product), or from a general perspective, (e.g. the demand limit value of all the products).

\subsection{Execution}

After creating the alert criteria, decision makers can use the Event Monitoring Systems to evaluate the situation with these criteria. This evaluation can be made automatically (e.g. by time intervals: hourly, daily or weekly) or manually. During these evaluations, the EMS-CE-PP checks the criteria (using the rules previously introduced) with the enterprise information, and as a result, events can be detected and decisions makers are alerted.

\subsection{Event impact classification}

Decision makers obtain new information after each execution. This information shows detected events related with each criterion and the impact level that produces that event. Also, the information about the number of occurrences of the event is stored to have historical information in order to obtain the likelihood and calculate the risk.

The impact of the event had been indicated previously by the expert and the likelihood is estimated by the system with the information of previous executions. This information allows decision makers to identify the impact of the event in order to evaluate the situation, try to solve the problem and, if necessary, change the ongoing production planning, and to obtain information about the event risks. 


\subsection{EMS-CE-PP Main Components Relationships}

This section shows the main components and their relationships included in this proposal.

An UML use case diagram identifies the interactions between an actor (role) and a system. In this case, a use case diagram has been included to show the relationship between the users (Expert and Production Planning Decision Maker) and the expected functionalities (figure 1).

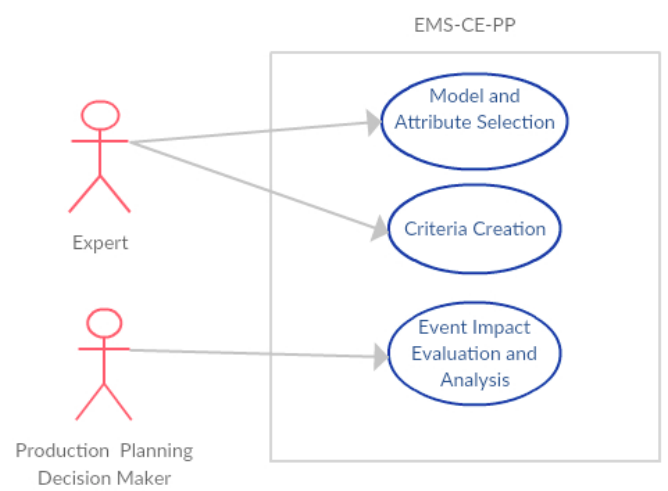

Fig. 1. EMS-CE-PP Use Case Diagram

Furthermore, figure 2 and figure 3 present the previous and the proposal situation. Figure 2 shows the initial situation where DSSs are used to make production planning decisions. Figure 3 displays the main components included in the event monitoring system framework proposed.



Fig. 2. DSSs used to make production planning (initial situation). 


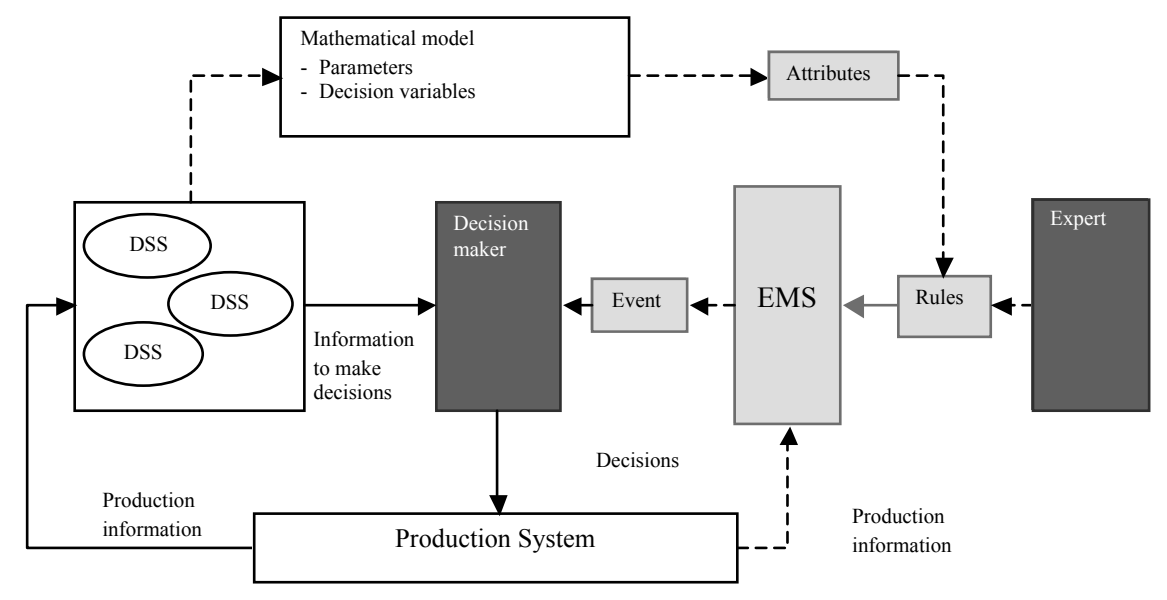

Fig. 3. The event monitoring system framework.

\section{EMS-CE-PP Prototype}

An Event Monitoring System prototype to Classify Events to reconsider the Production Planning was developed using Java libraries. The main elements used in the application were:

- Mathematical models used for the DSS to propose the production planning. The mathematical models have been defined in Mathematical Modeling Language (MPL).

- Databases with information about production. These databases include information about the current situation of the production system and the previous information of the production system when the DSS proposed the production planning.

- An internal database which includes the knowledge database.

The internal database has four main tables: attributes table to save the attributes of the model selected by the user; a criteria table, which stores the criteria created by decision makers; an execution criteria table, which saves information on execution (if execution is automatic or manually, interval time, etc.). Once execution has been run, the results are saved in the results table, which saves the information on each alert criterion (attributes values, event significance, event frequency, etc.).

\subsection{Model and Attributes Selection}

The scenario for this prototype is a company that generate its production planning with Model-Driven DSSs that use MPL (Mathematical Programming Language). MPL is an advanced modelling system that allows the model developer to formulate 
complicated optimization models in a clear, concise and efficient way. Models developed in MPL can then be solved with any of the multiple commercial optimizers available on the market today. MPL includes an algebraic modelling language that allows the model developer to create optimization models using algebraic equations [33]. Due to the fact that MPL is an structured language, it can be read easily for information systems. The figure 4 shows a basic example of an MPL file.

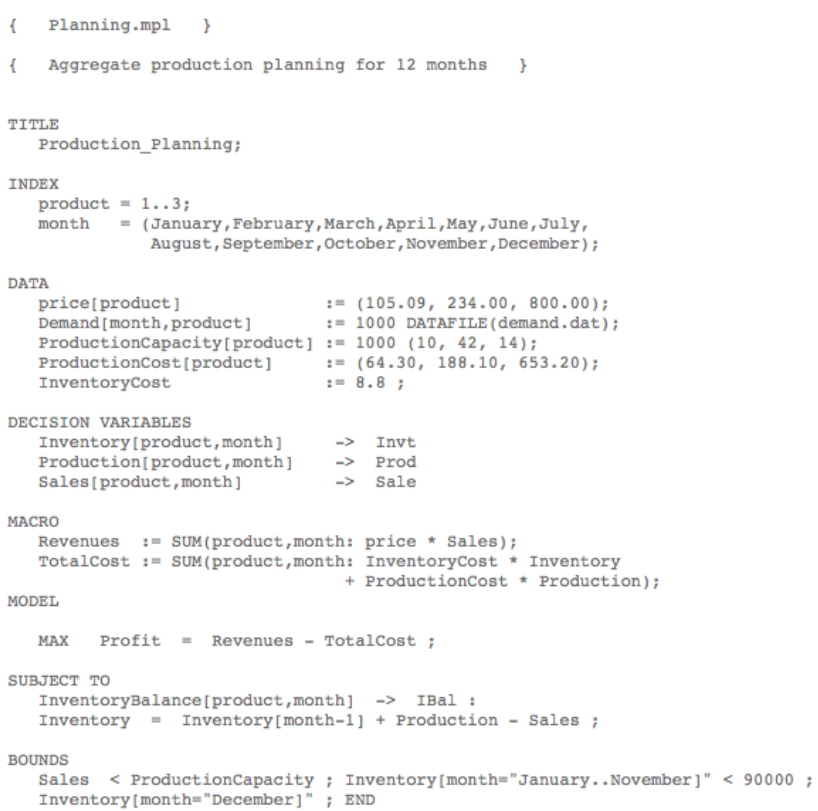

Fig. 4. Basic example of MPL file [33].

Every company can use its own set of MPL files, so it is possible to extract parameters and decision variables used in these models, and then, identifying those which can be affected by events.

The EMS prototype allows the user to select MPL files in order to load the attributes (parameters and decision variables) used in this model. Thus, the EMS prototype read the MPL file and identifies the parameters and decision variables included in the model. An expert can select between these attributes, which will be used to create the alert criteria. Furthermore, a link must be created between the attribute and the database (table and column) that contain their values. Figure 5 shows and example of selection of attributes, in this case, the attributes of "product" are showed. 


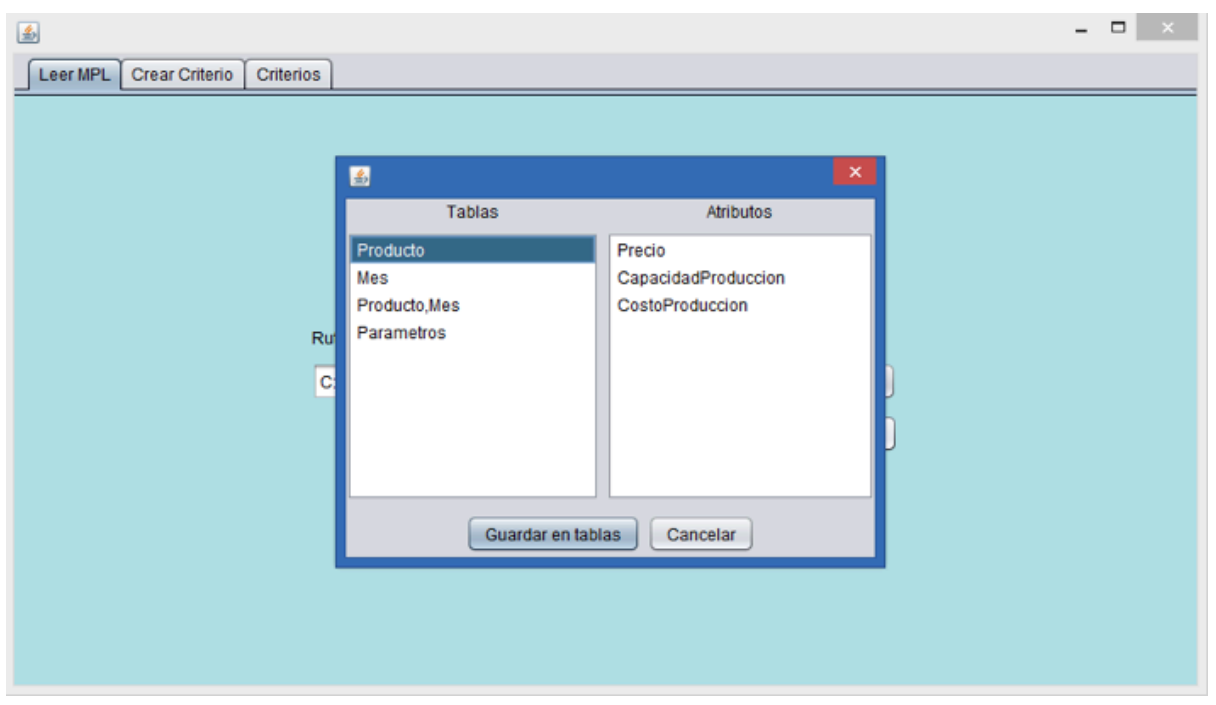

Fig. 5. Example of attribute selection.

The criteria creation form includes name, criteria operands, the logic operation to be performed with these operands, the impact level and a description. Also, some attribute characteristics need to be identified: (1) the attributes data in the criteria can be obtained from current values or previous values; (2) the alert criteria is general or for a particular object (Figure 6). This information is stored in the internal database with the set of criteria to be checked (Fig 7).

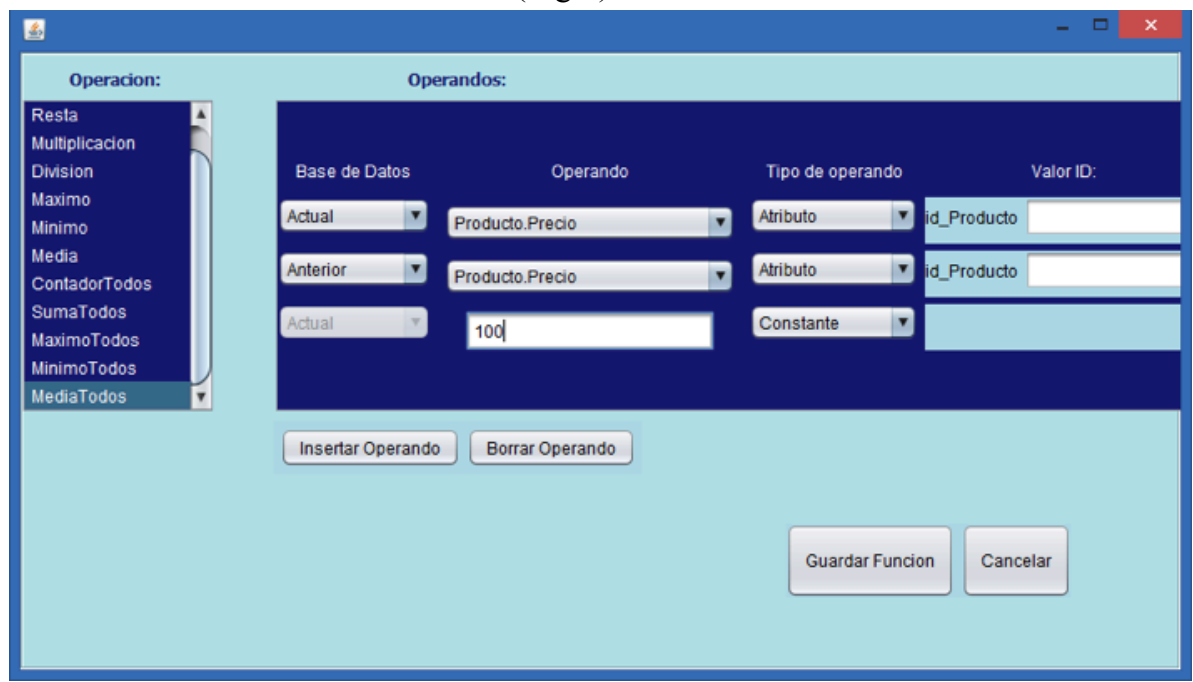

Fig. 6. Selection of the criteria operands. 


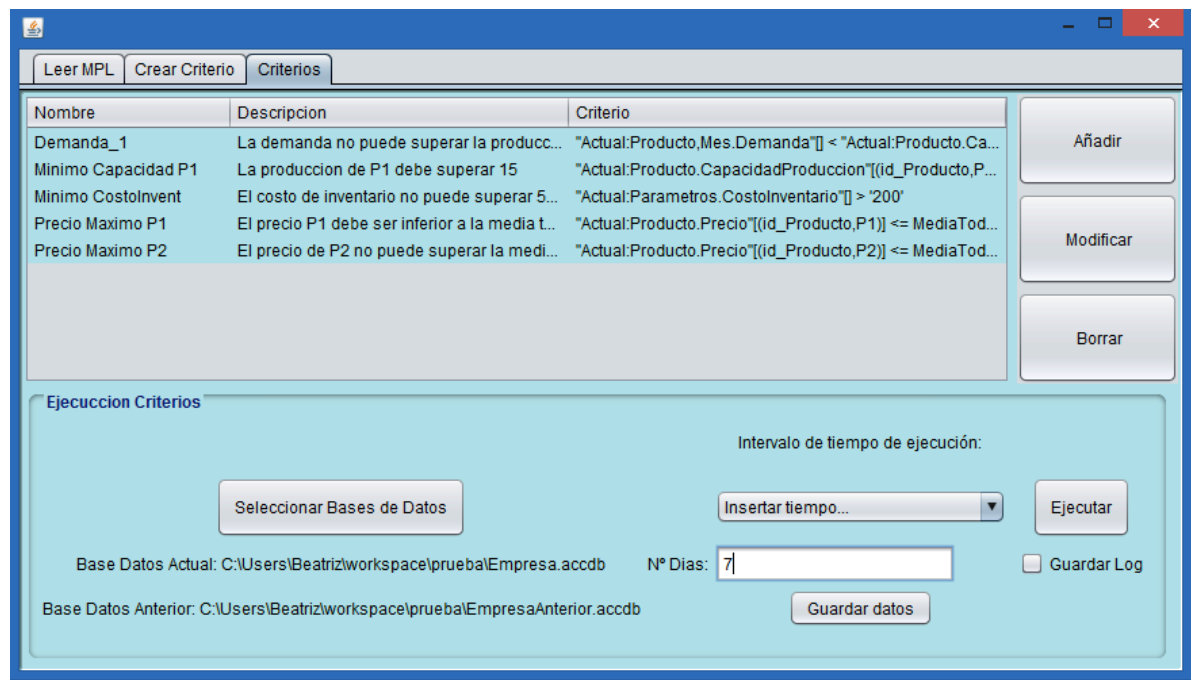

Fig. 7. Screen of the set of criteria included in the system

\subsection{Execution}

Periodical or manual monitoring can be made using the EMS-CE-PP prototype. The event monitoring system obtains information from the production databases in order to evaluate the criterion previously defined. This evaluation of each criterion allows identifying the impact levels for each criterion: Extremely Serious Level, Serious Level, Substantial Level, Moderate Level and Low Level. If an alarm appears in several levels for the same criterion, it is stored the most serious level.

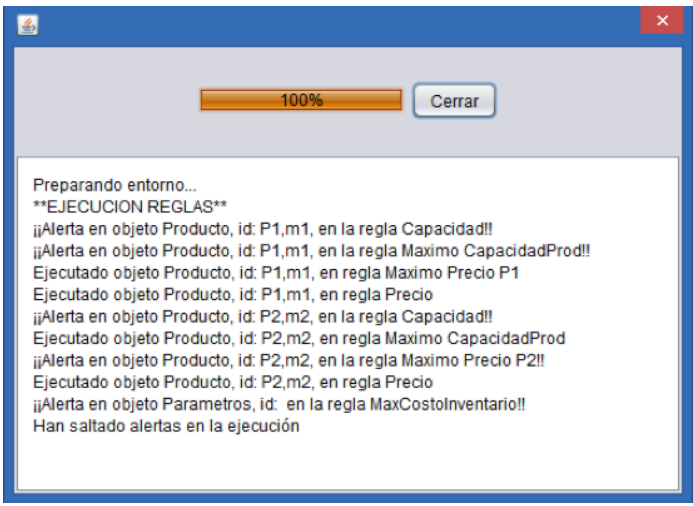

Fig. 8 Example of execution 


\subsection{Event Impact Classification}

The information is presented like a criterion list. A warning icon appears and indicates that an alarm occurs in this criterion. Production information is shown in white, yellow or red according to the impact level. This information can be evaluated for the decision-makers to reconsider the validity of the current production planning.

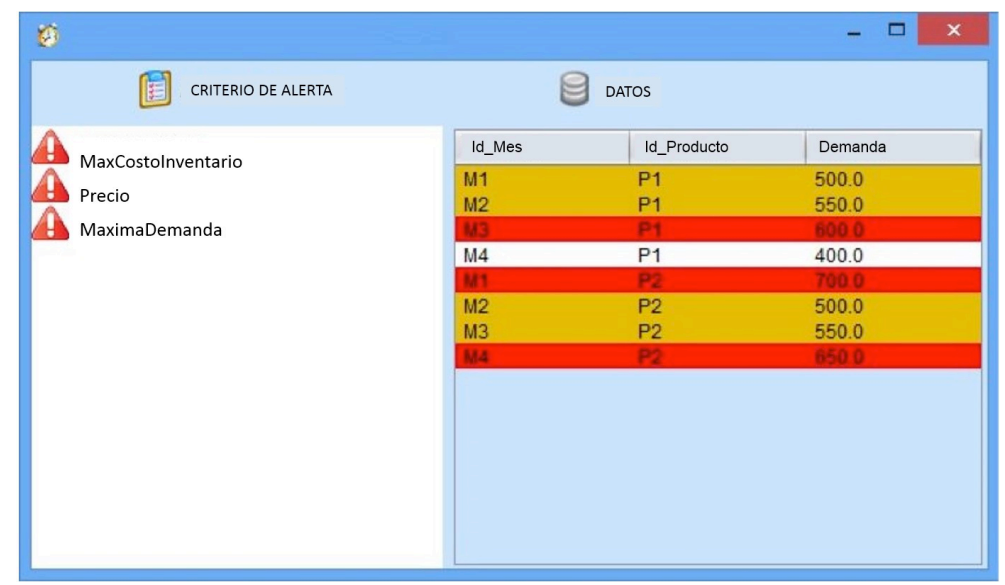

Figure 9. Example of Event Impact Classification

\section{Conclusions}

Production planning prepares the production area of the company for a future production scenario. This complex decision-making process requires an important volume of data and they can change when the production planning has been launched. Thus, unexpected events can appear while these plans are ongoing, which could have a major or minor impact on these ongoing plans. If the impact is major, it can force a change to be made in the established planning.

This research proposes an Event Monitoring Software Application based on an expert system to identify the events and to classify them according to their impact level on production planning. Experts with high knowledge about production planning can create production system alert criteria. In this way, decision makers can monitor these events and check if there are any unexpected events that impact in the ongoing production planning.

This proposal presents some advantages: i) own creation of impact criteria (rules) according to each production system to classify events; ii) connection with the DSS models used in the production planning and the production information system; iii), information to alert decision makers to decide whether to change production plans or not. 
An Event Monitoring System prototype to Classify Events and reconsider the Production Planning has been presented. The scenario for this prototype is a company that generate its production planning with Model-Driven DSSs that use MPL (Mathematical Programming Language).

A line for future research is to evaluate the economic impact of the events. A cost/benefits analysis could provide further information to the decision makers. Another area for future research is to identify the hierarchical decision levels in production planning and define different sets of criteria at each planning system level. Lastly, new Internet of Things and Sensor Technologies are able to provide further information about the production system. So, an Event Monitoring System could take these technologies into account in order to identify quickly relevant events in the Production System and to extend the EMS analysis with new information gathered with these technologies

\section{Acknowledgements}

This research has been carried out in the framework of the project GV/2014/010 funded by the Generalitat Valenciana (Identificación de la información proporcionada por los nuevos sistemas de detección accesibles mediante internet en el ámbito de las "sensing enterprises" para la mejora de la toma de decisiones en la planificación de la producción).

\section{References}

[1] R. Barták, On the Boundary of Planning and Scheduling: A Study. 1999.

[2] J. A. Buzacott, H. Corsten, R. Gössinger, and H. M. Schneider, Production Planning and Control: Basics and Concepts. Oldenbourg Wissenschaftsverlag, 2012.

[3] L. Özdamar, M. A. Bozyel, and S. I. Birbil, "A hierarchical decision support system for production planning (with case study)," Eur. J. Oper. Res., vol. 104, no. 3, pp. 403-422, Feb. 1998.

[4] W. Van Wezel, D. P. Van Donk, and G. Gaalman, "The planning flexibility bottleneck in food processing industries," J. Oper. Manag., vol. 24, no. 3, pp. 287-300, Apr. 2006.

[5] A. h. . Shamsuzzoha, S. Rintala, P. F. Cunha, P. S. Ferreira, T. Kankaanpää, and L. Maia Carneiro, "Event Monitoring and Management Process in a NonHierarchical Business Network," in Intelligent Non-hierarchical Manufacturing Networks, R. Poler, L. Carneiro, T. Jasinski, rc Zolghadri, and P. Pedrazzoli, Eds. John Wiley \& Sons, Inc., 2013, pp. 349-374.

[6] I. S. Sacala, M. A. Moisescu, and D. Repta, "Towards the development of the future internet based enterprise in the context of cyber-physical systems," 19th Int. Conf. Control Syst. Comput. Sci. CSCS 2013, pp. 405-412, 2013. 
[7] K. C. Chen, "Decision Support System for Tourism Development: System Dynamics Approach,” J. Comput. Inf. Syst., vol. 45, no. 1, pp. 104-112, 2004.

[8] A. Boza, M. M. E. Alemany, E. Vicens, and L. Cuenca, "Event Management in Decision-Making processes with Decision Support Systems," 5th Int. Conf. Comput. Commun. Control, 2014.

[9] S.-H. Liao, "Expert system methodologies and applicationsâ€"a decade review from 1995 to 2004," Expert Syst. Appl., vol. 28, no. 1, pp. 93-103, 2005.

[10] ISO, “73: 2009: Risk management vocabulary,” Int. Organ. Stand., 2009.

[11] F. T. S. Chan, K. C. Au, and P. L. Y. Chan, "A decision support system for production scheduling in an ion plating cell," Expert Syst. Appl., vol. 30, no. 4, pp. 727-738, 2006

[12] L. Weinstein and C.-H. Chung, "Integrating maintenance and production decisions in a hierarchical production planning environment," Comput. Oper. Res., vol. 26, no. 10â€“11, pp. 1059-1074, 1999.

[13] T. C. Poon, K. L. Choy, F. T. S. Chan, and H. C. W. Lau, "A real-time production operations decision support system for solving stochastic production material demand problems," Expert Syst. Appl., vol. 38, no. 5, pp. 4829-4838, 2011.

[14] M. M. Baron and M. E. Pate-Cornell, "Designing Risk-Management Strategies for Critical Engineering Systems," IEEE Trans. Eng. Manag., vol. 46, no. 1, pp. 87-100, 1999.

[15] SAP AG, "SAP AG 2014. Next-Generation Business and the Internet of Things. Studio SAP | 27484enUS (14/03).," 2014.

[16] L. M. Carneiro, P. Cunha, P. S. Ferreira, and A. Shamsuzzoha, "Conceptual Framework for Non-hierarchical Business Networks for Complex Products Design and Manufacturing," Procedia CIRP, vol. 7, pp. 61-66, 2013.

[17] A. Vargas, L. Cuenca, A. Boza, I. Sacala, and M. Moisescu, "Towards the development of the framework for inter sensing enterprise architecture," $J$. Intell. Manuf., Mar. 2014.

[18] G. Barash, C. Bartolini, and L. Wu, "Measuring and improving the performance of an IT support organization in managing service incidents," 2007, pp. 11-18.

[19] C. Bartolini, C. Stefanelli, and M. Tortonesi, "SYMIAN: Analysis and performance improvement of the IT incident management process," IEEE Trans. Netw. Serv. Manag., vol. 7, no. 3, pp. 132-144, Sep. 2010.

[20] R. Liu, A. Kumar, and W. van der Aalst, "A formal modeling approach for supply chain event management," Decis. Support Syst., vol. 43, no. 3, pp. 761-778, 2007.

[21] A. Söderholm, "Project management of unexpected events," Int. J. Proj. Manag., vol. 26, no. 1, pp. 80-86, 2008.

[22] L. A. Bearzotti, E. Salomone, and O. J. Chiotti, “An autonomous multi-agent approach to supply chain event management," Int. J. Prod. Econ., vol. 135, no. 1, pp. 468-478, 2012.

[23] M. M. Baron and M. E. Pate-Cornell, "Designing Risk-Management 
Strategies for Critical Engineering Systems," IEEE Trans. Eng. Manag., vol. 46 , no. 1 , pp. $87-100$.

[24] C. Bartolini, C. Stefanelli, and M. Tortonesi, "SYMIAN: Analysis and performance improvement of the IT incident management process," IEEE Trans. Netw. Serv. Manag., vol. 7, no. 3, pp. 132-144.

[25] L. A. (Tony) Cox Jr., "What's Wrong with Risk Matrices?," Risk Anal. An Int. J., vol. 28, no. 2, pp. 497-512, 2008.

[26] J. P. Shim, M. Warkentin, J. F. Courtney, D. J. Power, R. Sharda, and C. Carlsson, "Past, present, and future of decision support technology," Decis. Support Syst., vol. 33, no. 2, pp. 111-126, 2002.

[27] D. M. Steiger, "Enhancing User Understanding in a Decision Support System: A Theoretical Basis and Framework," http://dx.doi.org/10.1080/07421222.1998.11518214, 2015.

[28] E. Turban, J. Aronson, and T.-P. Liang, Decision Support Systems and Intelligent Systems 7 Edition. Pearson Prentice Hall, 2005.

[29] E. Turban and P. R. Watkins, Integrating Expert Systems and Decision Support Systems, vol. 10. 1986.

[30] D. Cohen and E. Asín, Sistemas de información para los negocios: un enfoque de toma de decisiones. McGraw-Hill, 2001.

[31] A. Boza, B. Cortés, M. M. E. Alemany, and E. Vicens, "Event Monitoring Software Application for Production Planning Systems," in Enhancing Synergies in a Collaborative Environment, 2014.

[32] A. Boza, F. Alarcón, M. M. E. Alemany, and L. Cuenca, "Event Classification System to Reconsider the Production Planning," in Proceedings of the 18th International Conference on Enterprise Information Systems, 2016, pp. 82-88.

[33] Maximal Software, "What is MPL?," 2016. [Online]. Available: http://www.maximalsoftware.com/mpl/what.html. 